\title{
Profiles of differentially expressed genes and overexpression of NEBL indicates a positive prognosis in patients with colorectal cancer
}

\author{
XIAO QIU, JUE-RONG FENG, FAN WANG, PENG-FEI CHEN, XIAO-XING CHEN, \\ RUI ZHOU, YING CHANG, JING LIU and QIU ZHAO \\ Department of Gastroenterology, Zhongnan Hospital of Wuhan University, \\ The Hubei Clinical Center and Key Laboratory of Intestinal and Colorectal Diseases, Wuhan, Hubei 430071, P.R. China
}

Received September 19, 2017; Accepted October 27, 2017

DOI: $10.3892 / \mathrm{mmr} .2017 .8210$

\begin{abstract}
The present study aimed to identify differentially expressed genes (DEGs) in colorectal cancer (CRC) and provide novel prognostic biomarkers for CRC. The microarray dataset GSE41258 was used to screen DEGs of CRC. Subsequently, a protein-protein interaction network of DEGs and Gene Ontology analysis were performed to identify hub genes and associated biological processes. Nebulette (NEBL) and complement C1q like 1 (C1QL1) were validated using reverse transcription-quantitative polymerase chain reaction in patients with CRC. Survival analysis was performed for the two hub genes. GSE41258 dataset included 182 CRC samples and 54 normal tissues. A total of 759 DEGs, including 279 upregulated and 480 downregulated were screened between both groups. NEBL and C1QL1 were identified as the two hub genes and upregulated genes involved in various biological processes, including 'regulation of biological quality' and 'response to stimulus', respectively. Additionally, the overexpression of NEBL and C1QL1 in experimental validation was consistent with the aforementioned bioinformatics analysis results. Survival analysis suggested that overexpressed NEBL in patients with CRC was associated with a positive prognosis for overall survival. In conclusion, CRC was associated with a large group of DEGs. From the upregulated genes, overexpressed NEBL in patients CRC indicated a positive prognosis
\end{abstract}

Correspondence to: Professor Qiu Zhao, Department of Gastroenterology, Zhongnan Hospital of Wuhan University, The Hubei Clinical Center and Key Laboratory of Intestinal and Colorectal Diseases, 169 Donghu Road, Wuhan, Hubei 430071, P.R. China

E-mail: zhaoqiu9090@126.com

Abbreviations: CRC, colorectal cancer; DEG, differentially expressed gene

Key words: colorectal cancer, differentially expressed genes, bioinformatics analysis, nebulette, prognosis for overall survival and may be used as a prognostic biomarker for patients with CRC.

\section{Introduction}

Colorectal cancer (CRC) is one of the most commonly diagnosed malignancies with an estimated 1.4 million cases and 693,900 mortalities by GLOBOCAN 2012 and a leading cause of cancer-associated mortality worldwide (1). The pathogenesis of CRC involving genetic and environmental factors, and their interactions has been investigated previously (2-4), and numerous risk factors of $\mathrm{CRC}$, such as tobacco use, unhealthy diet, obesity and physical inactivity, have been identified (1). It is widely recognized that loss of genomic stability and alterations in tumor suppressor genes and oncogenes have a key role in occurrence and progression of CRC $(2,5,6)$. Preventive measures, such as maintaining a healthy body weight, being physically active, minimizing consumption of red, processed meat and alcohol, and avoidance of smoking, screening options (fecal test, colonoscopy, stool DNA test, computed tomography), and improved treatments at an early stage of the disease are most likely attributed to reducing the CRC mortality rate observed in a large number of countries worldwide $(1,7)$. Additionally, advances in prognostic biomarkers that may allow personalized treatments could have also contributed to improvements in overall survival of patients with CRC. In addition, various biomarkers that may be used to assist in the diagnosis of CRC have been identified $(8,9)$. However, survival outcomes of CRC, particularly predictive and prognostic biomarkers, remain to be elucidated $(10,11)$. Therefore, valid prognostic biomarkers are urgently required to assist the prediction of the outcomes of CRC, which would result in earlier performances of preventative interventions or surgery that would improve survival rates (12).

The advent of high-throughput transcriptomic profiling, has allowed biomarker identification to be taken to the genomic level $(13,14)$. Additionally, it is important to understand the mRNA expression profiles involved in CRC and identify reliable biomarkers that may predict the survival of patients with CRC. The development of gene microarray and RNA sequencing has allowed for the use of gene expression 
profiling to identify genes associated with the carcinogenesis and development of CRC (13). Therefore, screening differentially expressed genes (DEGs) based on microarray analysis is an important novel way to investigate the pathogenesis of $\mathrm{CRC}$, additionally it is a quicker and more effective method of identification of the gene transcripts involved in energy metabolism in CRC and the mRNA isoforms used for diagnosis (15). Additionally, various databases, such as The Cancer Genome Atlas (TCGA) and PROGgeneV2 also help identify additional, valuable information using the data mining method.

In the current study, microarray analysis combined with a protein-protein interaction network was performed to identify the DEGs associated with CRC. Additionally, nebulette (NEBL) and complement Clq like 1 (C1QL1) were identified as hub genes of interest among DEGs using bioinformatics analysis. Subsequently, NEBL and C1QL1 were validated using reverse transcription-quantitative polymerase chain reaction (RT-qPCR) in $3 \mathrm{CRC}$ cell lines and $30 \mathrm{CRC}$ tissues with paired adjacent non-cancerous controls from patients. In order to investigate their prognostic values, the overall survival of patients with NEBL and C1QL1 in patients with CRC was determined using PROGgeneV2. Overall, the present study aimed to identify prognostic biomarkers among DEGs for $\mathrm{CRC}$ and allow for earlier treatment strategy intervention.

\section{Materials and methods}

Data preprocessing and DEGs screening. The mRNA expression profile dataset GSE41258 was downloaded from the Gene Expression Omnibus (GEO) database (www.ncbi. nlm.nih.gov/geo/), which was performed on the platform of Affymetrix Human Genome U133A Array. GSE41258 dataset contained 182 samples isolated from patients with CRC and 54 normal colorectal tissues (16). Expression profiling microarrays were firstly preprocessed by background correction and normalization, subsequently, the DEGs were statistically analyzed in both groups. All analyses were conducted using the limma package in $\mathrm{R}$ (17). The following thresholds were used to identify DEGs: $\mathrm{P}<0.01$ and $\mid \log _{2}$ fold-change $(\mathrm{FC}) \mid$ $>1$.

Construction of PPI network of DEGs and identification of hub genes. Protein-protein interactions (PPIs) are indirectly reflected as reciprocal interactions among DEGs. In the present study, the online server Search Tool for the Retrieval of Interacting Genes (STRING v10.5) was used to investigate these interactions (string-db.org). interacting DEGs were subsequently visualized using Cytoscape v3.4.0 software (18) to identify the hub genes, which were identified as highly connected genes in the interaction network if they had an interaction degree (interD) $>20$. In the present study, NEBL and C1QL1 were identified as hub genes, whose association with CRC remains unclear.

Gene Ontology $(G O)$ analysis of NEBL and C1QL1. In order to obtain additional insight into the functional enrichment of NEBL and C1QL1, GO analysis was performed. The Database for Annotation, Visualization, and Integrated Discovery (DAVID; david.ncifcrf.gov) version 6.8 was used to investigate the relevant biological meaning of the hub genes of interest.
Table I. Characteristics of patients with colorectal cancer in microarray and validation experiments.

\begin{tabular}{|c|c|c|c|}
\hline Characteristics & Microarray (n) & Validation (n) & P-value \\
\hline Age (years) & & & 0.074 \\
\hline Mean \pm SD & $63.30 \pm 13.98$ & $59.33 \pm 13.27$ & \\
\hline Range & 19-87 & $22-87$ & \\
\hline Gender & & & 0.156 \\
\hline Female & 86 & 10 & \\
\hline Male & 96 & 20 & \\
\hline AJCC stage & & & 0.002 \\
\hline I & 28 & 3 & \\
\hline II & 48 & 16 & \\
\hline III & 49 & 11 & \\
\hline IV & 57 & 0 & \\
\hline \multicolumn{4}{|l|}{ TNM stage } \\
\hline Tumor & & & 0.518 \\
\hline $\mathrm{T} 1$ & 4 & 1 & \\
\hline $\mathrm{T} 2$ & 34 & 3 & \\
\hline $\mathrm{T} 3$ & 131 & 25 & \\
\hline $\mathrm{T} 4$ & 13 & 1 & \\
\hline Node & & & 0.245 \\
\hline N0 & 93 & 18 & \\
\hline N1 & 46 & 9 & \\
\hline $\mathrm{N} 2$ & 43 & 3 & \\
\hline Metastasis & & & $<0.01$ \\
\hline M0 & 125 & 30 & \\
\hline M1 & 57 & 0 & \\
\hline
\end{tabular}

SD, standard deviation; AJCC, American Joint Committee on Cancer; TNM, tumor-node-metastasis.

A P-value $<0.05$ indicated a statistically significant functional annotation.

Cell lines and cell culture. The HCT116, HT29, DLD1 human CRC cell lines and the NCM460 normal human colorectal epithelial cell line were used in this study. All the cell lines were purchased from the Type Culture Collection of the Chinese Academy of Sciences (Shanghai, China). These cell lines were cultured in Dulbecco's modified Eagle's medium (GE Healthcare Life Sciences, Logan, UT, USA) containing $10 \%$ fetal bovine serum (Gibco; Thermo Fisher Scientific, Inc., Waltham, MA, USA). All cells were cultured at $37^{\circ} \mathrm{C}$ in a humidified atmosphere containing $95 \% \mathrm{O}_{2}$ and $5 \% \mathrm{CO}_{2}$.

Clinical sample collection and ethical approval. Fresh CRC tissue samples and paired adjacent non-cancerous tissue samples were obtained after surgical resection prior to radiation or chemotherapy from 30 patients at the Department of General Surgery, Zhongnan Hospital of Wuhan University (Wuhan, China) from March 2017 to June 2017. The samples were frozen and stored in liquid nitrogen. The morphological classification of the tumor was performed according to the American Joint Committee on Cancer (AJCC) staging 


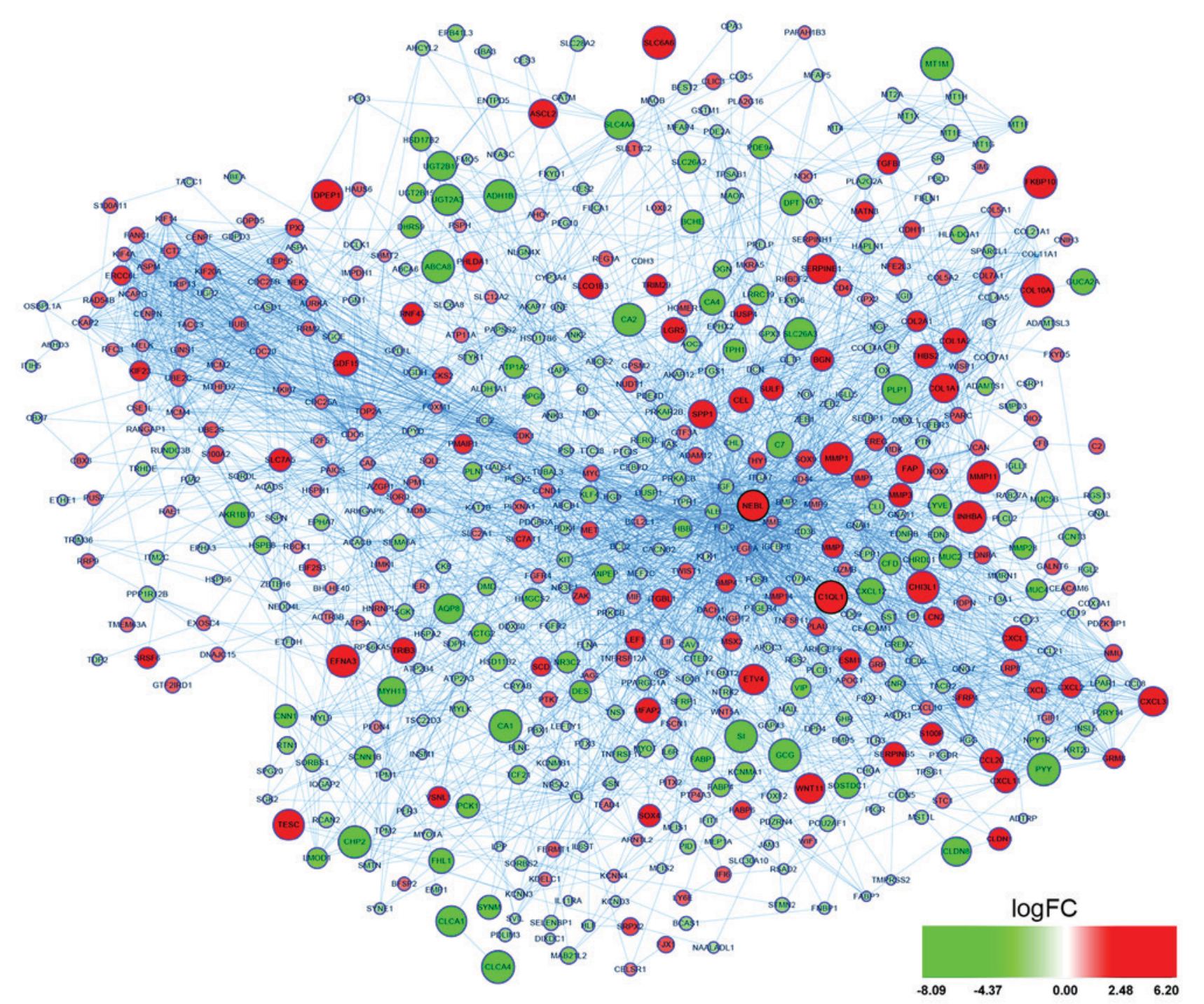

Figure 1. Protein-protein interaction network of total DEGs in colorectal cancer. Green nodes represent downregulated genes, red nodes represent upregulated genes. The size of the nodes is proportional to the expression level of DEGs. NEBL and C1QL1 (black circle outline) were the two hub genes based on highly connected degree and upregulated DEGs. DEGs, differentially expressed genes; NEBL, nebulette; C1QL1, complement Clq like 1.

system (19). Written informed consent was obtained from all patients. The study was approved by the Ethics Committee of Zhongnan Hospital of Wuhan University. All procedures performed in studies involving human participants were in accordance with the ethical standards of the Ethical Committee of Zhongnan Hospital of Wuhan University, and with the principle of the 1964 Helsinki declaration and its later amendments or comparable ethical standards. The sample characteristics for CRC patients are presented in Table I.

$R N A$ extraction and $R T-q P C R$. Total RNA was extracted from the cells and tissues using TRIzol reagent (Invitrogen; Thermo Fisher Scientific, Inc.) according to the manufacturer's protocol. RNA quantification was performed using a spectrophotometer (NanoDrop 2000, Thermo Fisher Scientific, Inc.). Total RNA $(1 \mu \mathrm{g})$ was used with a first-strand cDNA using a synthesis kit (Thermo Fisher Scientific, Inc.) to perform the RT reaction $\left(65^{\circ} \mathrm{C}\right.$ for $5 \mathrm{~min}, 42^{\circ} \mathrm{C}$ for $60 \mathrm{~min}$ and $70^{\circ} \mathrm{C}$ for $5 \mathrm{~min}$ ). The mRNA expression levels of the selected genes were subsequently evaluated by qPCR using a QuantStudio ${ }^{\mathrm{TM}}$
6 Flex Real-Time PCR instrument (Thermo Fisher Scientific, Inc.) with SYBR Premix Ex Taq ${ }^{\mathrm{TM}}$ II mix (Takara Bio, Inc., Otsu, Japan). The qPCR reaction was performed with an initial denaturation step of $95^{\circ} \mathrm{C}$ for 5 min followed by 40 cycles of $95^{\circ} \mathrm{C}$ for $3 \mathrm{sec}$ and $61^{\circ} \mathrm{C}$ for $30 \mathrm{sec}$. The NEBL primers were forward (F) 5'-GGAATGCAAGCTGGCACTGACA-3' and reverse (R) 5'-GAGTGTCTGTGCTCACCTGCAT-3'; C1QL1 F 5'-AGTATGTGGGCAGACCTCTGCA-3' and R 5'-CCA GCTTGATGAAGACCTCGTC-3'; and GAPDH F 5'-AGA AGGCTGGGGCTCATTTG-3' and R 5'-GCAGGAGGCATT GCTGATGAT-3'. The relative mRNA expression levels were calculated using the $2^{-\Delta \Delta \mathrm{Cq}}$ method (20). GAPDH was used as the internal control.

Statistical analysis. All analyses of experimental validation were performed using SPSS version 17.0 (SPSS, Inc., Chicago, IL, USA). Student's t-test was used for comparisons between groups. Differences between the CRC cell lines and control cell line were analyzed using one-way analysis of variance with a Bonferroni correction. $\mathrm{P}<0.05$ was considered to indicate 


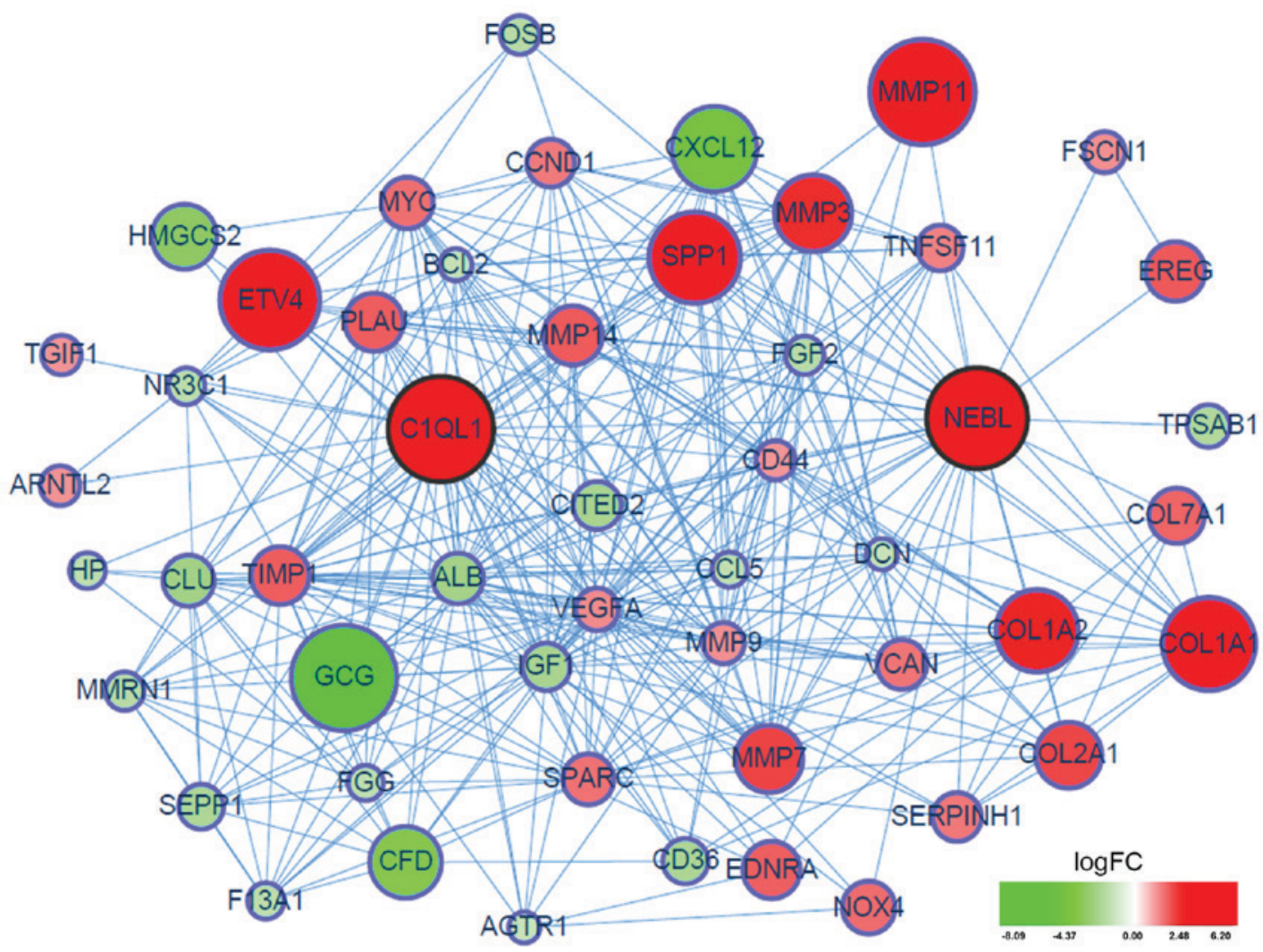

Figure 2. Protein-protein interaction network of the two hub genes (NEBL and C1QL1) and linker genes in colorectal cancer. Green nodes represent downregulated genes, red nodes represent upregulated genes. The size of the nodes is proportional to the expression level. NEBL, nebulette; C1QL1, complement Clq like 1.

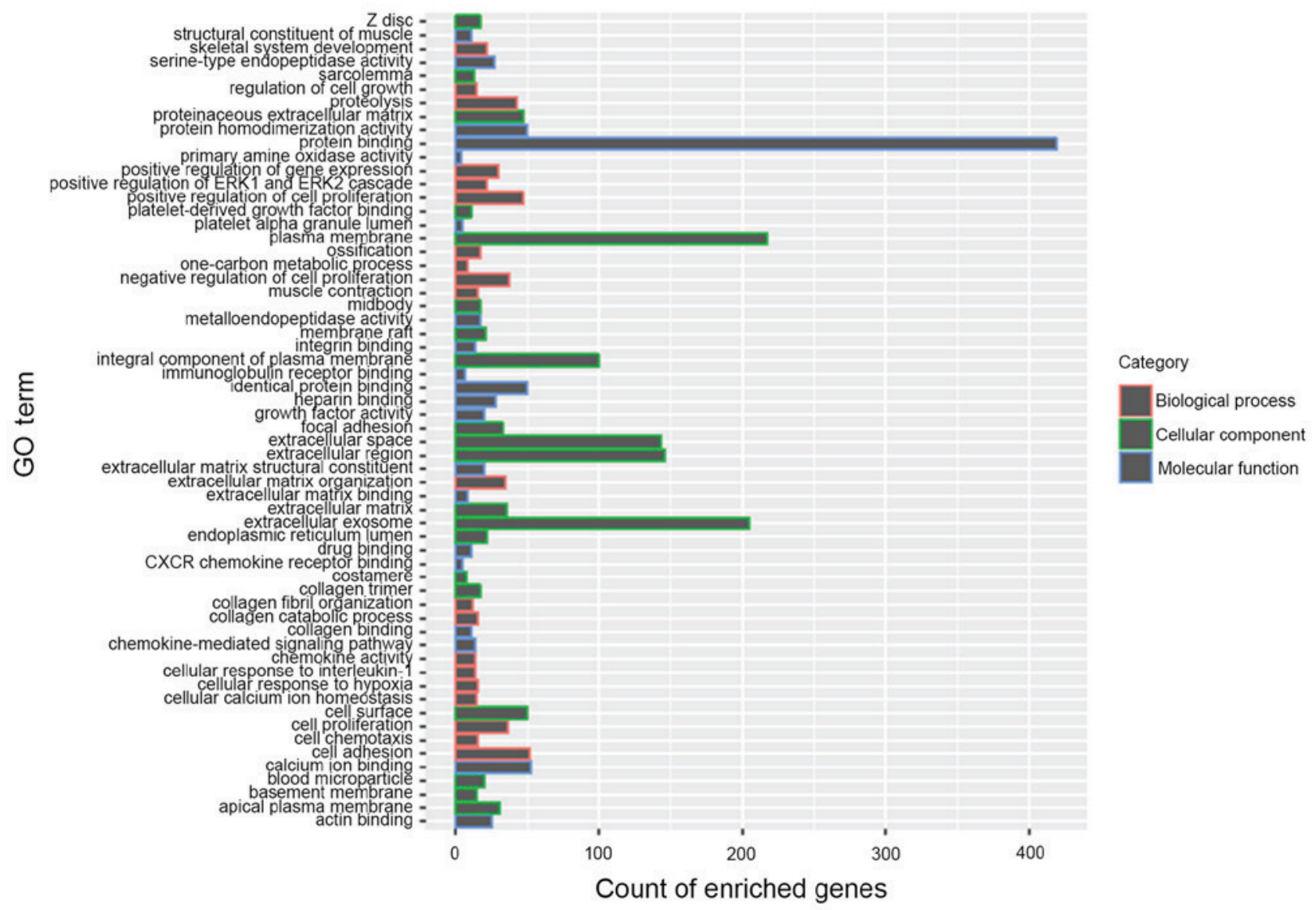

Figure 3. Gene Ontology analysis of differentially expressed genes. 
Table II. Hub genes with their representative significantly enriched terms of GO biological processes.

\section{A, NEBL}

\begin{tabular}{|c|c|c|}
\hline GO ID & Description & P-value \\
\hline 65008 & Regulation of biological quality & $5.79 \times 10^{-9}$ \\
\hline 51128 & $\begin{array}{l}\text { Regulation of cellular component } \\
\text { organization }\end{array}$ & $1.57 \times 10^{-5}$ \\
\hline 65007 & Biological regulation & $1.37 \times 10^{-4}$ \\
\hline 90066 & $\begin{array}{l}\text { Regulation of anatomical } \\
\text { structure size }\end{array}$ & $4.28 \times 10^{-4}$ \\
\hline 33043 & Regulation of organelle organization & $4.98 \times 10^{-4}$ \\
\hline 50789 & Regulation of biological process & $3.71 \times 10^{-3}$ \\
\hline
\end{tabular}

B, C1QL1

\begin{tabular}{lll}
\hline GO ID & \multicolumn{1}{c}{ Description } & P-value \\
\hline 50896 & Response to stimulus & $2.74 \times 10^{-7}$ \\
7626 & Locomotory behavior & $1.56 \times 10^{-6}$ \\
7610 & Behavior & $1.92 \times 10^{-6}$ \\
\hline
\end{tabular}

NEBL, nebulette; C1QL1, complement C1q like 1.

a statistically significant difference. Data are presented as mean \pm standard deviation. Experiments were repeated independently at least three times.

Survival analysis of NEBL and C1QL1. The median value of each hub gene in tumor samples was calculated. Samples with expression higher than the median value were placed in the high expression group, and the samples with expression lower than the median value were placed in the low expression group. Survival analysis was performed using PROGgeneV2 tool for both groups (21), which allowed the investigation of prognostic implications of gene expression associated with CRC in the corresponding microarray datasets. The GSE41258 dataset was used to predict the overall survival of the hub genes of interest in the present study. In addition, survival models were adjusted for multiple covariates such as age, gender and cancer stages. The survival curves were visualized using Kaplan-Meier plots for the high and low expression groups. $\mathrm{P}<0.05$ was considered to indicate a statistically significant difference.

\section{Results}

DEGs screening and identification of hub genes. A total of 759 DEGs were identified, including 279 upregulated and 480 downregulated DEGs in primary CRC samples compared with normal colon samples (Fig. 1). By constructing PPI network of DEGs, NEBL and C1QL1 were identified as the two hub genes based on highly connected degree (interD $=34$ and 33, respectively) as well as being overexpressed DEGs in the GSE41258 dataset (Fig. 1). Additionally, a PPI network of the two hub genes and their linker genes in CRC was presented in Fig. 2.
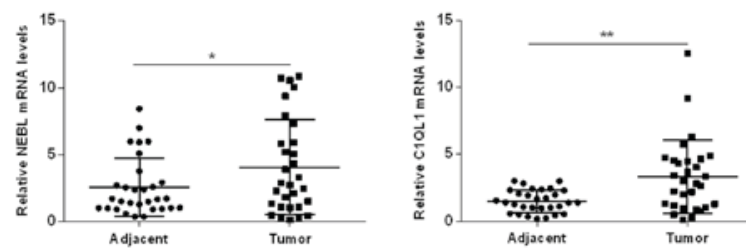

B
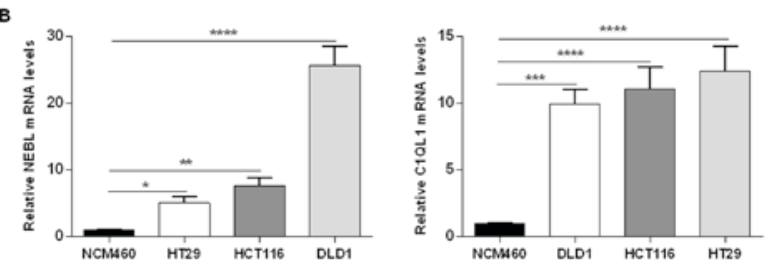

Figure 4. mRNA expression of NEBL and C1QL1 in CRC samples. (A) Relative mRNA levels of NEBL and C1QL1 in 30 CRC patient tissues. (B) Expression levels in the 3 CRC cell lines were detected by RT-qPCR. Each experiment was repeated independently at least three times. Data were presented as the mean \pm standard deviation. ${ }^{*} \mathrm{P}<0.05,{ }^{* *} \mathrm{P}<0.01,{ }^{* * * *} \mathrm{P}<0.001$, ${ }^{* * * * *} \mathrm{P}<0.0001$ vs. adjacent/NCM460 control group. CRC, colorectal cancer; NEBL, nebulette; C1QL1, complement C1q like 1.

GO analysis of NEBL and C1QL1. Functional enrichment map of DEGs, including NEBL and C1QL1 was presented in Fig. 3, which represents a holistic view of the functions of all screened DEGs. In addition, under the threshold of $\mathrm{P}<0.05$, the biological processes NEBL was significantly enriched in were 'regulation of biological quality', 'regulation of cellular component organization', 'biological regulation', 'regulation of anatomical structure size', 'regulation of organelle organization' and 'regulation of biological process'. The biological processes C1QL1 was enriched in were 'response to stimulus', 'locomotory behavior' and 'behavior' (Table II).

Validation of NEBL and CIQL1 mRNA expression. The mRNA expression levels of NEBL and C1QL1 in the present study were confirmed in the tissues of patients with CRC and CRC cell lines using RT-qPCR. The mRNA expression levels of NEBL and C1QL1 were higher in CRC tissues when compared with normal adjacent tissues (NEBL, $\mathrm{P}=0.049$; C1QL1, P=0.001; Fig. 4A). NEBL and C1QL1 expression levels were upregulated in the $3 \mathrm{CRC}$ cell lines when compared with NCM460 cell line (Fig. 4B).

Prognosis of NEBL and C1QL1. Survival analysis was performed for NEBL and C1QL1 using PROGgeneV2 based on the GSE41258 dataset. In the overall dataset, 91 samples were demonstrated to exhibit a high expression of NEBL, and a further 91 samples were demonstrated to exhibit a low expression of NEBL. During $>16$ years of follow up, the incidence of death in high and low expression groups was 42 and 60 events, respectively. The median survival of high and low expression of NEBL were 3,600 and 2,580 days, respectively, which indicated that the overexpression of NEBL in patients with CRC was significantly associated with a positive prognosis $[\mathrm{P}=0.019$; hazard ratio (HR), $0.75 ; 95 \%$ confidence interval (CI), 0.59-0.95; Fig. 5A]. Additionally, the prognosis of NEBL was associated with age and gender (Table III). However, the expression of C1QL1 was not associated with the prognosis of CRC ( $\mathrm{P}=0.504$; HR, 1.6; 95\% CI, 0.41-6.29; Fig. 5B). 
Table III. Overexpression of NEBL and C1QL1 associated with the prognosis of colorectal cancer using PROGgene version 2.0 and GSE41258 dataset.

\begin{tabular}{lccc}
\hline Survival models $^{\mathrm{a}}$ & NEBL & P-value & C1QL1 \\
\hline Overall & $0.75(0.59-0.95)$ & 0.02 & $1.60(0.41-6.29)$ \\
After adjustment for age & $0.75(0.59-0.95)$ & 0.02 & $1.91(0.47-7.69)$ \\
After adjustment for gender & $0.76(0.59-0.97)$ & 0.03 & $1.32(0.33-5.24)$ \\
After adjustment for clinical stage & $1.08(0.84-1.38)$ & 0.54 & $0.65(0.15-2.92)$ \\
After adjustment for TMN stage & $0.87(0.68-1.12)$ & 0.29 & $0.96(0.18-4.99)$ \\
\hline
\end{tabular}

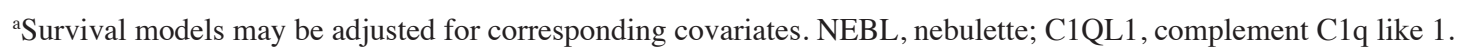
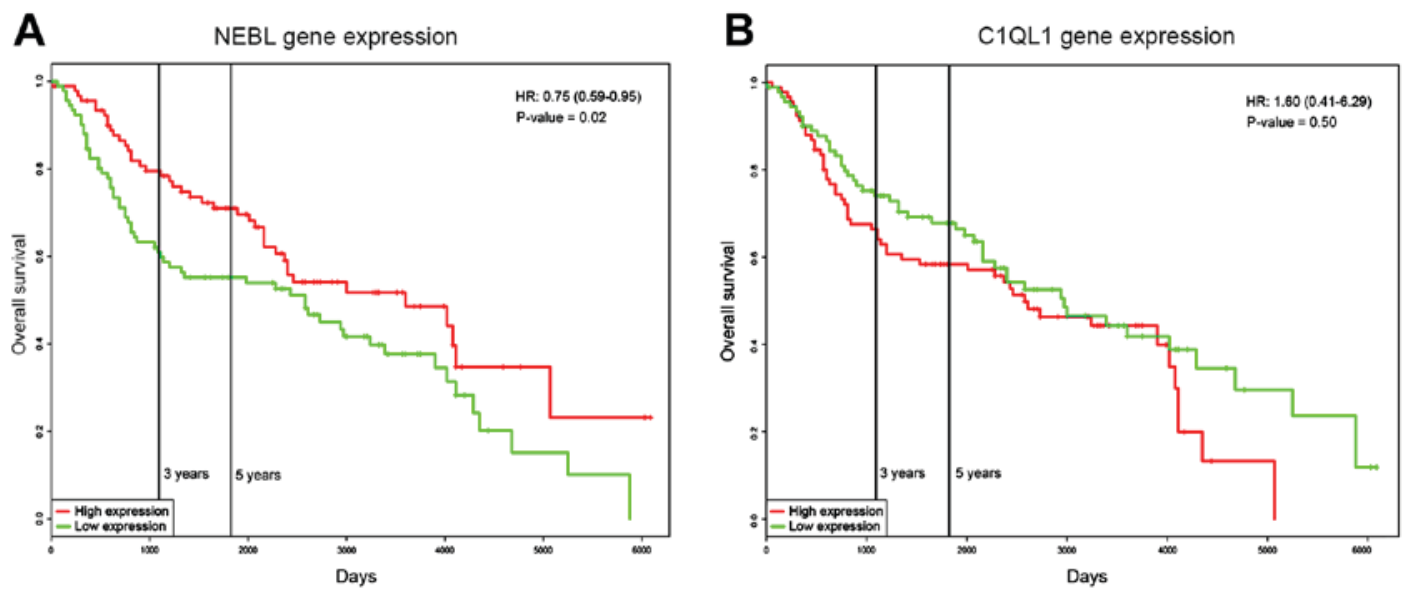

Figure 5. Kaplan-Meier survival plots for the expression of NEBL and C1QL1 in the overall survival of CRC patients. (A) Kaplan-Meier survival curve indicates the higher expression of NEBL is associated with longer the overall survival of patients with CRC. (B) Kaplan-Meier survival curve indicates that the expression of C1QL1 is not associated with the overall survival of patients with CRC patients. CRC, colorectal cancer; NEBL, nebulette; C1QL1, complement C1q like 1.

\section{Discussion}

CRC is one of the most frequent malignant tumors worldwide (1). The overall survival of patients with CRC remains poor, primarily due to the late diagnosis and metastasis (22). There are a few prognostic biomarkers identified at present that may be applied successfully in clinical practice; therefore, it is essential to identify novel potential biomarkers of prognosis in patients with CRC in order to facilitate early intervention.

The NEBL gene was previously reported to be highly expressed in cardiac muscle, regulating the attachment and migration of extracellular matrix (23). It was primarily identified as a novel mixed lineage leukemia fusion partner gene in an infant that suffered from acute myeloid leukemia (24). Additionally, NEBL has previously been demonstrated to have a significant effect on the regulation of cardiac function (25), concurrently its mutation may lead to various cardiomyopathies (26). NEBL was also identified to be associated with foot processes in podocyte injury and osteogenic abilities $(27,28)$. The aforementioned validated biological processes of NEBL were in accordance with the findings of the GO analysis performed in the present study. However, information regarding the expression and role of NEBL in tumors, particularly in $\mathrm{CRC}$ is insufficient. The present study revealed that NEBL was highly expressed in CRC tissues and cell lines. It is of note that the present study observed that higher NEBL expression was associated with improved patient prognosis. A prognostic biomarker refers to a measurable and quantifiable method that is closely associated with clinical outcomes in the certain context of patients. Therefore, the findings of the current study suggested that NEBL overexpression may be a prognostic biomarker for CRC. However, although NEBL was highly expressed in CRC, its effect on clinical phenotypes of CRC remains to be elucidated. Instead of acting as an oncogene, it may have an important role in combating oncogenes involved in CRC progression, and once a tumor develops it may be overexpressed in patients with CRC, thus contributing to an improved prognosis for CRC.

The C1QL1 gene was originally cloned as a senescence-associated gene that was highly expressed in the brain, which may also have a role in neuronal differentiation (29). It has been previously demonstrated that C1QL1 specifically binds to its receptor, the adhesion $\mathrm{G}$ protein-coupled receptor 3 , controlling the stereotyped pattern of connectivity and regulating maturation of synapses between climbing fibers and Purkinje cells $(30,31)$. However, its expression and role in CRC have remain to be investigated. To the best of our knowledge the present study was the first to demonstrate that C1QL1 was upregulated in CRC; however, it was unable to identify a significant correlation between C1QL1 expression and the prognosis of patients with CRC.

NEBL and C1QL1 have been investigated in various other diseases in the recent years $(25,26,31)$. The present study 
identified NEBL and C1QL1 to be involved in CRC using microarray analysis, where both DEGs were overexpressed in CRC tissues. Additionally, the experimental findings revealed were consistent among CRC cell lines and tissues and confirmed the outcomes of bioinformatics analysis. However, several limitations of the present study should be mentioned. Firstly, a larger sample size of CRC tissues with survival time are required in order to confirm the current conclusions. In addition, the biological function of the two hub genes remains unknown, and the relationship between them and the tumor malignant phenotype should be evaluated in future studies.

In conclusion, numerous DEGs including NEBL and C1QL1 were identified in CRC tissues, NEBL was overexpressed in CRC tissues and may be used as a prognostic biomarker for patients with CRC.

\section{Acknowledgements}

The present study was supported by a research grant from the National Natural Science Foundation of China (grant no. 81472735).

\section{References}

1. Torre LA, Bray F, Siegel RL, Ferlay J, Lortet-Tieulent J and Jemal A: Global cancer statistics, 2012. CA Cancer J Clin 65: 87-108, 2015.

2. Vogelstein B, Fearon ER, Hamilton SR, Kern SE, Preisinger AC, Leppert M, Nakamura Y, White R, Smits AM and Bos JL: Genetic alterations during colorectal-tumor development. N Engl J Med 319: 525-532, 1988.

3. Rahmani F, Avan A, Hashemy SI and Hassanian SM: Role of $\mathrm{Wnt} / \beta$-catenin signaling regulatory microRNAs in the pathogenesis of colorectal cancer. J Cell Physiol 233: 811-817, 2018.

4. Louis P, Hold GL and Flint HJ: The gut microbiota, bacterial metabolites and colorectal cancer. Nat Rev Microbiol 12 661-672, 2014.

5. Grady WM and Carethers JM: Genomic and epigenetic instability in colorectal cancer pathogenesis. Gastroenterology 135: 1079-1099, 2008.

6. Nakayama T,Hirakawa H, Shibata K,Nazneen A, Abe K, Nagayasu T and Taguchi T: Expression of angiopoietin-like 4 (ANGPTL4) in human colorectal cancer: ANGPTL4 promotes venous invasion and distant metastasis. Oncol Rep 25: 929-935, 2011.

7. Gellad ZF and Provenzale D: Colorectal cancer: National and international perspective on the burden of disease and public health impact. Gastroenterology 138: 2177-2190, 2010.

8. Ahmed FE: miRNA as markers for the diagnostic screening of colon cancer. Expert Rev Anticancer Ther 14: 463-485, 2014

9. Rawson JB and Bapat B: Epigenetic biomarkers in colorectal cancer diagnostics. Expert Rev Mol Diagn 12: 499-509, 2012.

10. Walther A, Johnstone E, Swanton C, Midgley R, Tomlinson I and Kerr D: Genetic prognostic and predictive markers in colorectal cancer. Nat Rev Cancer 9: 489-499, 2009.

11. Verdaguer H, Saurí T and Macarulla T: Predictive and prognostic biomarkers in personalized gastrointestinal cancer treatment J Gastrointest Oncol 8: 405-417, 2017.

12. van der Stok EP, Spaander MCW, Grünhagen DJ, Verhoef C and Kuipers EJ: Surveillance after curative treatment for colorectal cancer. Nat Rev Clin Oncol 14: 297-315, 2017.

13. Malapell U, Mayo de-Las-Casas C, Rocco D, Garzon M, Pisapia P, Jordana-Ariza N, Russo M, Sgariglia R, De Luca C, Pepe F, et al: Development of a gene panel for next-generation sequencing of clinically relevant mutations in cell-free DNA from cancer patients. Br J Cancer 116: 802-810, 2017.

14. Broderick P, Carvajal-Carmona L, Pittman AM, Webb E, Howarth K, Rowan A, Lubbe S, Spain S, Sullivan K, Fielding S, et al: A genome-wide association study shows that common alleles of SMAD7 influence colorectal cancer risk. Nat Genet 39: 1315-1317, 2007.
15. Snezhkina AV, Krasnov GS, Zaretsky AR, Zhavoronkov A, Nyushko KM, Moskalev AA, Karpova IY, Afremova AI, Lipatova AV, Kochetkov DV, et al: Differential expression of alternatively spliced transcripts related to energy metabolism in colorectal cancer. BMC Genomics 17 (Suppl 14): 1011, 2016.

16. Sheffer M, Bacolod MD, Zuk O, Giardina SF, Pincas H, Barany F, Paty PB, Gerald WL, Notterman DA and Domany E: Association of survival and disease progression with chromosomal instability: A genomic exploration of colorectal cancer. Proc Natl Acad Sci USA 106: 7131-7136, 2009.

17. Ritchie ME, Phipson B, Wu D, Hu Y, Law CW, Shi W and Smyth GK: Limma powers differential expression analyses for RNA-sequencing and microarray studies. Nucleic Acids Res 43: e47, 2015.

18. Shannon P, Markiel A, Ozier O, Baliga NS, Wang JT, Ramage D, Amin N, Schwikowski B and Ideker T: Cytoscape: A software environment for integrated models of biomolecular interaction networks. Genome Res 13: 2498-2504, 2003.

19. Hari DM, Leung AM, Lee JH, Sim MS, Vuong B, Chiu CG and Bilchik AJ: AJCC cancer staging manual 7th edition criteria for colon cancer: Do the complex modifications improve prognostic assessment? J Am Coll Surg 217: 181-190, 2013.

20. Livak KJ and Schmittgen TD: Analysis of relative gene expression data using real-time quantitative PCR and the 2(-Delta Delta C(sT)) method. Methods 25: 402-408, 2001.

21. Goswami CP and Nakshatri H: PROGgeneV2: Enhancements on the existing database. BMC Cancer 14: 970, 2014.

22. Keane MG and Johnson GJ: Early diagnosis improves survival in colorectal cancer. Practitioner 256: 15-18, 2, 2012.

23. Lee EJ, De Winter JM, Buck D, Jasper JR, Malik FI, Labeit S, Ottenheijm CA and Granzier H: Fast skeletal muscle troponin activation increases force of mouse fast skeletal muscle and ameliorates weakness due to nebulin-deficiency. PLoS One 8: e55861, 2013

24. Cóser VM, Meyer C, Basegio R, Menezes J, Marschalek R and Pombo-de-Oliveira MS: Nebulette is the second member of the nebulin family fused to the MLL gene in infant leukemia. Cancer Genet Cytogenet 198: 151-154, 2010

25. Mastrototaro G, Liang X, Li X, Carullo P, Piroddi N, Tesi C, Gu Y, Dalton ND, Peterson KL, Poggesi C, et al: Nebulette knockout mice have normal cardiac function, but show Z-line widening and up-regulation of cardiac stress markers. Cardiovasc Res 107: 216-225, 2015

26. Perrot A, Tomasov P, Villard E, Faludi R, Melacini P, Lossie J, Lohmann N, Richard P, De Bortoli M, Angelini A, et al: Mutations in NEBL encoding the cardiac Z-disk protein nebulette are associated with various cardiomyopathies. Arch Med Sci 12: 263-278, 2016

27. Miao J, Fan Q, Cui Q, Zhang H, Chen L, Wang S, Guan N, Guan Y and Ding J: Newly identified cytoskeletal components are associated with dynamic changes of podocyte foot processes. Nephrol Dial Transplant 24: 3297-3305, 2009.

28. Aino M, Nishida E, Fujieda Y, Orimoto A, Mitani A, Noguchi T, Makino H, Murakami S, Umezawa A, Yoneda T and Saito M: Isolation and characterization of the human immature osteoblast culture system from the alveolar bones of aged donors for bone regeneration therapy. Expert Opin Biol Ther 14: 1731-1744, 2014.

29. Bérubé NG, Swanson XH, Bertram MJ, Kittle JD, Didenko V, Baskin DS, Smith JR and Pereira-Smith OM: Cloning and characterization of CRF, a novel Clq-related factor, expressed in areas of the brain involved in motor function. Brain Res Mol Brain Res 63: 233-240, 1999.

30. Yuzaki M: The C1q complement family of synaptic organizers: Not just complementary. Curr Opin Neurobiol 45: 9-15, 2017.

31. Sigoillot SM, Iyer K, Binda F, González-Calvo I, Talleur M, Vodjdani G, Isope P and Selimi F: The secreted protein C1QL1 and its receptor bai3 control the synaptic connectivity of excitatory inputs converging on cerebellar Purkinje cells. Cell Rep pii: S2211-1247: 00059-00065, 2015.

This work is licensed under a Creative Commons Attribution-NonCommercial-NoDerivatives 4.0 International (CC BY-NC-ND 4.0) License. 